\title{
Role of the dorsal hippocampus in object memory load
}

\author{
Sara Sannino, ${ }^{1,2,6}$ Fabio Russo, ${ }^{1,2,6}$ Giulia Torromino, ${ }^{3,4}$ Valentina Pendolino, ${ }^{5}$ \\ Paolo Calabresi, ${ }^{5}$ and Elvira De Leonibus ${ }^{1,2,7}$ \\ ${ }^{1}$ Institute of Genetics and Biophysics (IGB), CNR, 80131, Naples, Italy; ${ }^{2}$ Telethon Institute of Genetics and Medicine (TIGEM), 80131, \\ Naples, Italy; ${ }^{3}$ Dipartimento di Biologia e Biotecnologie, Università degli Studi di Roma "La Sapienza," 00185, Rome, Italy; ${ }^{4}$ Centro di \\ Ricerca in Neurobiologia-D. Bovet, Università degli Studi di Roma "La Sapienza," 00185, Rome, Italy; ${ }^{5}$ Fondazione Santa Lucia, \\ Istituto di Ricovero e Cura a Carattere Scientifico, 00143, Rome, Italy
}

\begin{abstract}
The dorsal hippocampus is crucial for mammalian spatial memory, but its exact role in item memory is still hotly debated. Recent evidence in humans suggested that the hippocampus might be selectively involved in item short-term memory to deal with an increasing memory load. In this study, we sought to test this hypothesis. To this aim we developed a novel behavioral procedure to study object memory load in mice by progressively increasing the stimulus set size in the spontaneous object recognition task. Using this procedure, we demonstrated that naive mice have a memory span, which is the number of elements they can remember for a short-time interval, of about six objects. Then, we showed that excitotoxic selective lesions of the dorsal hippocampus did not impair novel object discrimination in the condition of low memory load. In contrast, the same lesion impaired novel object discrimination in the high memory load condition, and reduced the object memory span to four objects. These results have important heuristic and clinical implications because they open new perspective toward the understanding of the role of the hippocampus in item memory and in memory span deficits occurring in human pathologies, such as Alzheimer's disease and schizophrenia.
\end{abstract}

[Supplemental material is available for this article.]

The dorsal hippocampus-HP (the CA fields, dentate gyrus, and subicular complex) is generally crucial for mammalian memory, but its exact role in memory formation is still hotly debated around a conspicuous number of rival theories. Although, in the early 1980s, Olton and colleagues (Wible et al. 1992; Wan et al. 1994) showed that HP lesions produced an impairment on both spatial and nonspatial memory independently on the retention time interval, converging evidence presented a dichotomous view between spatial and item memory (Brown and Aggleton 2001), and between short-term (STM) and long-term (LTM) memory (Shrager et al. 2008), regarding the role of the HP. Brown and Aggleton (2001) proposed that the HP is essential for associational, multi-item, re-collective aspects of recognition memory, but has little or no involvement for single item recognition, which would be mediated by the perirhinal cortex. Squire and colleagues (Broadbent et al. 2004), in contrast, suggested that the HP is selectively involved in LTM, and not in STM or working memory (WM), independently of the nature of the information.

Studies in rodents unequivocally demonstrated that HP lesions impair memory consolidation of newly acquired spatial information, and quite consistently affect spatial STM, thus suggesting that the HP is involved in spatial memory independently of the retention interval (Hunsaker et al. 2008; Vago and Kesner 2008). Much more controversial is the role of HP in item memory. HP lesions generally have no or minor effect on item STM and LTM (Broadbent et al. 2004; Ainge et al. 2006; Hunsaker et al. 2008). Nevertheless, recent accumulating experi-

\footnotetext{
${ }^{6}$ These authors contributed equally to this work.

${ }^{7}$ Corresponding author.

E-mail elvira.deleonibus@igb.cnr.it.

Article is online at http://www.learnmem.org/cgi/doi/10.1101/lm.025213.111.
}

mental evidence suggests that the HP is indeed involved in item STM when the memory load is increased by the number of items (Cave and Squire 1991; Beason-Held et al. 1999; Levy et al. 2003, 2004; Shrager et al. 2008; van Vugt et al. 2010). Item or digit memory after short-retention interval is, in fact, affected in patients or monkeys with HP damage and in amnesic patients in the condition of high load (Cave and Squire 1991; Levy et al. 2003), and HP gamma oscillations increase as humans perform a WM task with increasing number of faces or letters (van Vugt et al. 2010). The role of HP in mediating item memory only when the memory load increases is suggesting a possible neural substrate specifically activated to increase the memory capacity, independently of the nature of incoming information (item vs. spatial). However, this role of the HP in mediating item memory at short-retention interval in conditions of high load is not predicted by spatial and memory consolidation theories of HP and by the extra-hippocampal medial temporal lobe structures' involvement in item recognition (Brown and Aggleton 2001; Shrager et al. 2008). Furthermore, it has not been confirmed in rodent studies yet. In fact, there is only one study that, to our knowledge, addressed this issue, reporting that extensive lesion of the HP in rats did not affect the performance of an odor span task (Dudchenko et al. 2000).

Unfortunately, the paucity of behavioral procedures for rodents to study item memory load does not help to solve this issue, which is relevant not only to better clarify the role of the HP in memory, but also to understand how the brain faces a high memory load. This is an almost unexplored field, although it has an extremely high clinical relevance since the memory span is, indeed, selectively affected in many neurologic and psychiatric conditions, such as aging, Alzheimer's and Parkinson's disease, and schizophrenia (Brebion 2003; Clatworthy et al. 2009; Fischer et al. 2010; Lee et al. 2010). The aim of this study was, therefore, to set up a novel behavioral procedure to study object memory 
span in mice, to verify how many different objects mice can retain for a short-retention time, and what is the exact role of the dorsal HP in object memory span.

\section{Results}

\section{A novel behavioral procedure to study object memory span in mice}

Recognition memory is a process by which a subject identifies a stimulus as previously experienced (Steckler et al. 1998) and/or the ability to discriminate stimuli of different familiarity, i.e., the discrimination between novel and familiar stimuli. The spontaneous object recognition task is widely used in rodents to study recognition memory (Broadbent et al. 2004; Ainge et al. 2006). In its classical version, experimental subjects are first exposed to two identical objects and subsequently, after a predetermined delay, tested for their ability to discriminate between familiar and new objects (Broadbent et al. 2004). Thus, the memory load of this task is very low (one object to remember).

In this study, we modified the spontaneous object recognition task to develop a behavioral procedure for the analysis of object memory span in mice. Consistent with previously reported studies in humans, primates, and rodents, we augmented the memory load increasing the stimulus set size (Fig. 1) and tested new object discrimination in animals subjected to three, four, six, or nine Different Objects Task (DOT) (Fig. 1A-D). As a control task, we used the Identical Objects Task (IOT) (Fig. 1E-H) (see Materials and Methods), in which we increased the number of identical objects, which is supposed to not increase the amount of information to be processed. In rodents, unlike humans and nonhuman primates, objects' related information is acquired through a direct physical exploration of the objects, and this requires time. Nevertheless, an excess or a lack of study time might

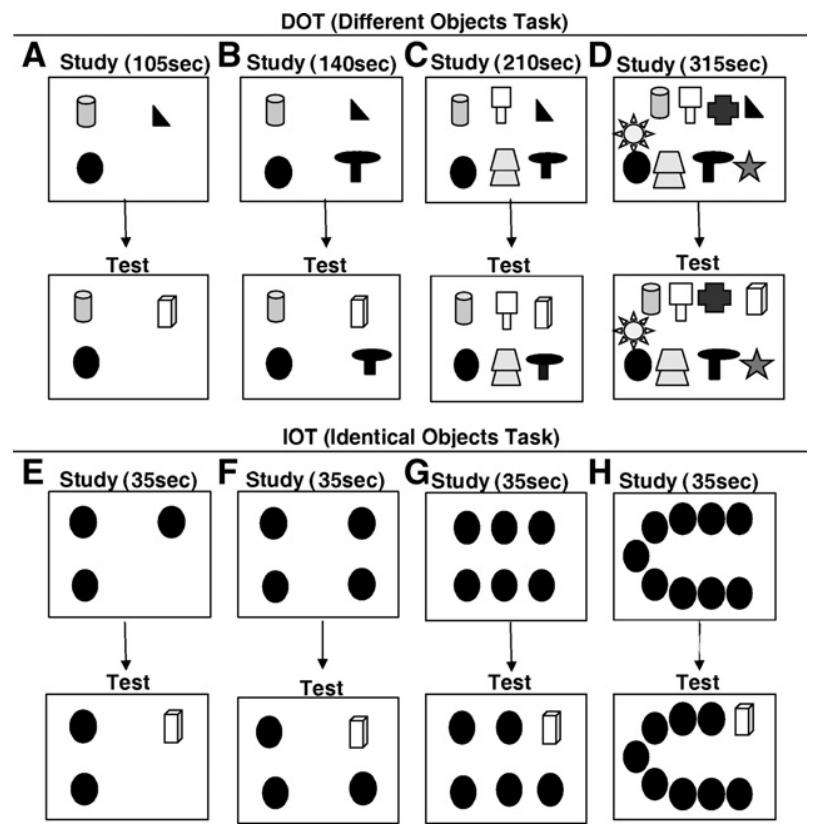

Figure 1. Schematic representation of the study and test phase of the object memory span task. During the study phase, animals were exposed to the $(A-D)$ 3-DOT, 4-DOT, 6-DOT, and 9-DOT (Different Objects Task) objects or (E-H) IOT (Identical Objects Task) objects. The figure reports the object exploration time (in seconds) the animals were allowed to accumulate during the study phase. During the test phase, only one object was substituted with a different one in all conditions.

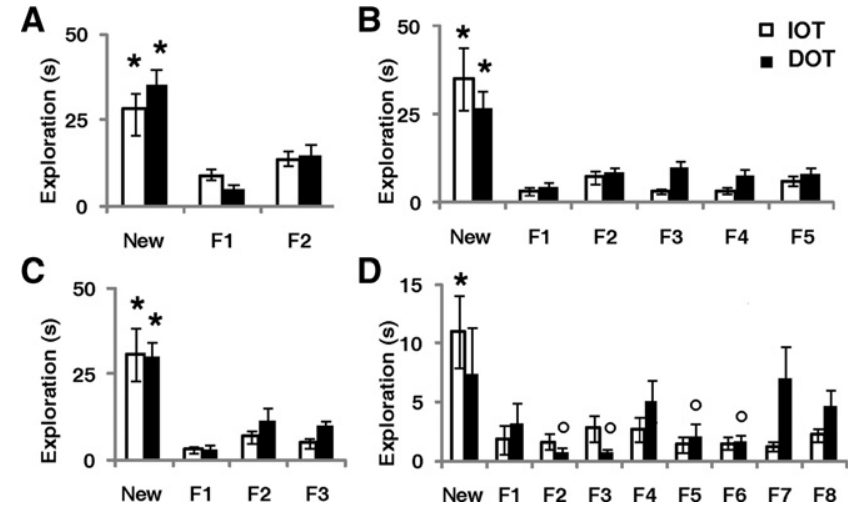

Figure 2. Naive mice have a limited object memory span. Histograms report new and familiar (F) objects exploration during the 5-min test phase, performed at 1-min retention interval, of the object memory span task, in naive animals. The new object was explored significantly more than all of the familiar objects in the IOT (white bar), independently of the number of objects presented $(A-D)$. The same behavior was observed in the DOTs (black bar) with three, four, and six different objects $(A-C)$. (D) In contrast, with nine different objects, animals did not explore the new object significantly more than four of the familiar objects (F1, F4, F7, F8). (*) $P<0.05$ new versus all of the other familiar objects; $\left({ }^{\circ}\right) P<0.05$ new versus each of the familiar objects; Duncan post hoc tests.

have been a cause of learning deficit or of overlearning. Following the logical hypothesis that increasing the number of different but not identical objects would have increased the memory load, we calculated the study time for each experimental condition. We supposed that in the IOT, increasing the number of identical samples would not have increased the memory load, and therefore the same study time might have been sufficient to solve the task. Indeed, we found (Experiment 1) that object discrimination, after 1 min of delay (STM), occurred in the IOT (Fig. 2A-D) independently of the number of objects presented, and although we gave $35 \mathrm{sec}$ of total exploration during the study phase (Table 1). In the IOT, the new object was explored significantly more than all the other familiar objects. We also hypothesized that the same study time was not sufficient to solve the Different Objects Tasks, because the amount of information to acquire was increased and therefore required the animal to spend more time in contact with each of the different objects. Based on this, we demonstrated (Experiment 1) that when the study time allowed was proportional to the number of different objects to explore, the animals could discriminate up to six different objects (Fig. 2A-C). However, this finding did not exclude the possibility that if we gave less study time (e.g., the same amount given in the IOT), animals could not solve the DOT. Experiment 2 tested this possibility and clearly demonstrated that limiting the study time to $35 \mathrm{sec}$ in animals exposed to three or six different objects (Experiment 2), as we did for the IOT, impaired object discrimination (Fig. 3A,B). The demonstration that, for a good performance during the test phase, the study time in the DOT had to be proportional to the number of different items clearly suggests, in our opinion, that increasing the stimulus set size requires more time to be processed, which can be explained only in terms of increased memory load. Nevertheless, there is a limit to the number of different objects mice could discriminate. Despite the proportional increase in the study time, in the 9-DOT (Fig. 2D), the new object was explored significantly more than only four of the familiar objects (F2, F3, F5, F6), suggesting that the number of objects presented was too high for the animals' memory spans. A discrimination impairment in the 9-DOT cannot be attributed to an insufficient study time, or fatigue effect, because they spent an average of 
Table 1. Mean total objects and mean object exploration during the study phase for Experiment 1

\begin{tabular}{|c|c|c|c|c|c|c|}
\hline Experiment & $\begin{array}{c}\text { Number } \\
\text { of } \\
\text { objects }\end{array}$ & Task & RI & $\begin{array}{c}\text { Total } \\
\text { exploration } \\
\text { study phase } \\
\text { (sec } \pm \text { SEM) }\end{array}$ & $\begin{array}{l}\text { Mean object } \\
\text { exploration } \\
(\text { sec } \pm \text { SEM })\end{array}$ & \\
\hline \multirow[t]{7}{*}{1} & 3 & DOT & 1 min & $82 \pm 9.2$ & $27.3 \pm 3.1$ & 9 \\
\hline & 4 & DOT & $1 \mathrm{~min}$ & $108 \pm 8.4$ & $27 \pm 2.1$ & \\
\hline & & IOT & $1 \mathrm{~min}$ & $28 \pm 3.6$ & $7.1 \pm 0.1$ & \\
\hline & 6 & DOT & $1 \mathrm{~min}$ & $165 \pm 14$ & $27.5 \pm 2.3$ & 8 \\
\hline & & IOT & $1 \min$ & $35 \pm 0.4$ & $5.9 \pm 0.06$ & \\
\hline & 9 & DOT & $1 \mathrm{~min}$ & $225 \pm 24$ & $25 \pm 2.7$ & 10 \\
\hline & & IOT & $1 \mathrm{~min}$ & $32 \pm 2$ & $3.5 \pm 0.2$ & 11 \\
\hline
\end{tabular}

The table reports (from left to right) the experiment to which the data refer, the number of objects used, the kind of task (Different Objects Task [DOT] or Identical Objects Task [IOT]), the retention interval (RI), the total exploration time accumulated during the study phase, the mean object exploration (total amount exploration/number of objects), and the number of subjects per group.

$25 \pm 2.7$ sec on each of the nine different objects, which is perfectly comparable to the value recorded in the 3-DOT, 4-DOT, and 6-DOT (Table 1). The selective impairment in the new object discrimination in the 9-DOT (Fig. 2D) suggests that mice have an object memory span and that in CD1 outbred mice it is about six different objects, which is similar to that of human subjects (Luck and Vogel 1997).

\section{Role of the dorsal hippocampus in object memory load}

Previous studies in nonhuman and human primates with HP lesions demonstrated that the HP selectively mediates item memory at short retention time intervals only in high memory load conditions (Beason-Held et al. 1999; Levy et al. 2003, 2004). The behavioral procedure we described in Experiment 1 was designed to parallel studies in human amnesics in which novel and familiar items are simultaneously compared (Beason-Held et al. 1999; Broadbent et al. 2004; Ainge et al. 2006; Shrager et al. 2008). Therefore, we used it to address the role of the dorsal HP in the object memory load. To this aim, six different groups of sham or HP lesioned animals were tested in the three, four, or six object tasks (both identical and different in a randomized order), using the same behavioral procedure described above. Figure 4, A and B, shows a representative picture of a coronal section of animals injected within the dorsal HP with vehicle (Sham) or neurotoxic concentrations (Les) of $N$-methyl-D-aspartate (NMDA). The lesion we performed was restricted to the dorsal part of HP (Fig. 4B; Supplemental Fig. S1). The behavioral results clearly showed that the lesion did not affect object exploration during the study phase (Table 2). They also showed that a dorsal HP lesion did not affect novel object discrimination after $1 \mathrm{~min}$ when the animals were exposed to the 3-DOT or 4-DOT (Fig. 4C,D), but it strongly impaired it when the animals were exposed to the 6-DOT (Fig. 4E). Furthermore, no impairment was observed in the IOT (Fig. 4F-H). This demonstrates that dorsal HP lesion impairs novel object discrimination at $1 \mathrm{~min}$ of delay depending on the stimulus set size.

According to a previous study, $\sim 50 \%-75 \%$ reduction of total HP volume is sufficient to impair spatial memory, while an almost complete damage (75\%-100\% total HP volume reduction) is necessary to impair object LTM (Broadbent et al. 2004). A qualitative re-construction of the average extension of the lesion we per- formed (Supplemental Fig. S1) indicates that we destroyed $\sim 50 \%-75 \%$ of HP volume according to this study (Broadbent et al. 2004); therefore, it should have spared object LTM in low memory load conditions, but impaired spatial memory. To test this hypothesis, two further groups of sham and HP lesioned animals were submitted to the 3-DOT and tested after $24 \mathrm{~h}$ (Experiment 4). Lesioned animals, as well as sham animals, explored the new object significantly more than the familiar ones, suggesting an intact object LTM at low memory load conditions (Fig. 5A).

To test whether the same lesion was, instead, effective in impairing spatial memory, we subjected some of the animals tested in the object tasks to the water maze task (Experiment 5) using a behavioral procedure previously described for CD1 mice (Sargolini et al. 2003). Sham and lesioned-HP animals did not differ in their escape latency when the platform was visible $\left(F_{(1,19)}=0.8\right.$; $P=0.4$ ) and approachable using a taxon strategy. In contrast, when the platform was submerged (the day after), we found that lesioned animals had significantly higher escape latency as compared with sham animals $\left(F_{(1,19)}=6.3 ; P=0.02\right)$ during training and did not prefer the target annulus during the probe (Fig. $5 \mathrm{~B}, \mathrm{C})$, suggesting that the dorsal HP lesion we performed selectively impaired spatial learning and memory in the water maze. All together, the results of this lesion study demonstrate that lesion of the dorsal HP does not impair novel object discrimination in a condition of low memory load independently of the memory retention interval, but strongly impaired novel object discrimination at 1 min of delay in a high memory load condition, as well as spatial memory formation.

Nevertheless, a careful analysis of the histological data (Supplemental Fig. S1) showed that in many animals, the lesion extended toward the cortical areas surrounding the HP, namely, the parietal cortex. To exclude the possibility that the impairment we observed in the 6-DOT was not due to the spread of toxin to these cortical areas, two further groups of animals (Experiment 6) were injected with saline (sham) or NMDA (lesioned) exactly as done to perform the HP lesion, with the only difference of using a more dorsal injection coordinate (see Materials and Methods). This lesion spared the HP and lesioned the cortical areas surrounding it (Fig. 6A,B). We then subjected the animals to the 6-DOT and 6-IOT as previously described and found no effect of the lesion on the study (Table 3 ) or the test phase (Fig. 6C,D). This result,

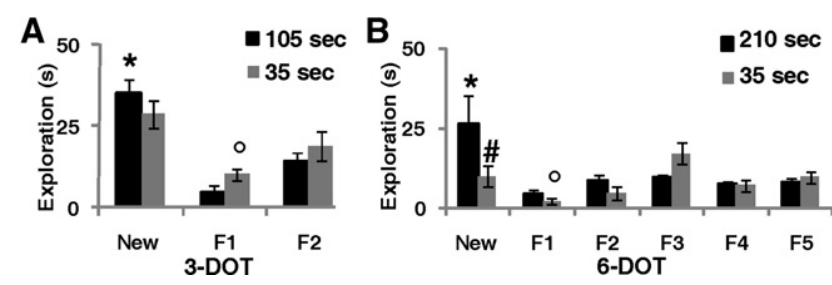

Figure 3. The Different Objects Task requires more study time to be performed. Histograms report new and familiar (F) object exploration during the 5-min test phase, in naive animals allowed, during the study phase, to explore three $(A)$ and six $(B)$ different objects for a total of 35 sec. Their performance was compared with that of animals allowed to explore for a total of 105 or $210 \mathrm{sec}$, respectively. When the exploration time allowed during the study phase was reduced to $35 \mathrm{sec}$, animals in the 3-DOT explored the new object significantly more than one of the two familiar (F1) objects $(A)$. In the 6-DOT $(B)$, the group allowed to explore for $35 \mathrm{sec}$ explored the new object significantly more than the familiar one (F1) and similarly to all the other five familiar objects (F2, F3, F4, F5); furthermore, new object exploration in this group was reduced as compared with the group allowed to explore for $210 \mathrm{sec}$ during the study phase. $\left(^{*}\right) P<0.05$ new versus all of the other familiar objects; $\left({ }^{\circ}\right)$ $P<0.05$ new versus each of the familiar objects; (\#) $P<0.05$ for 35 versus 105 or 210; within object; Duncan post hoc tests. 
A

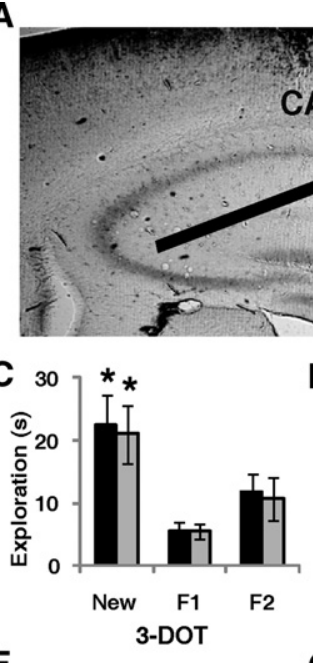

$\mathbf{F}$

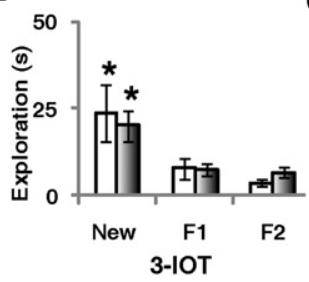

B
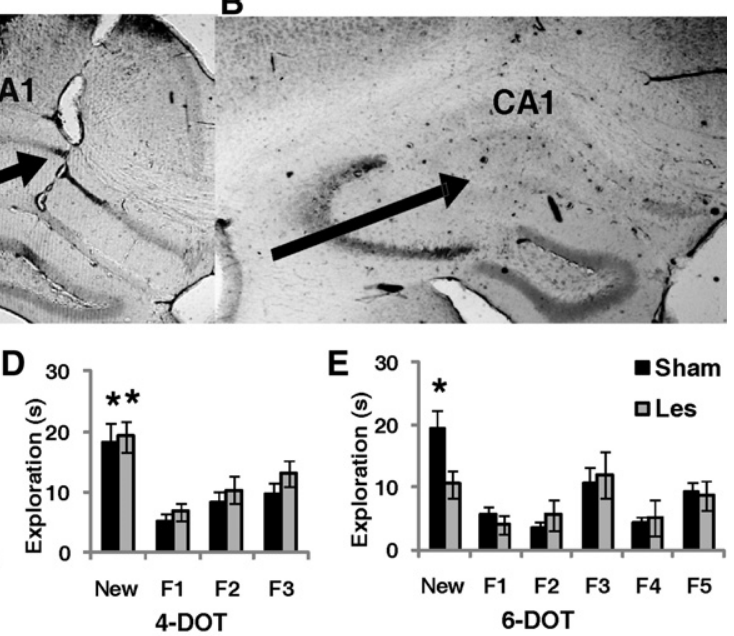

G

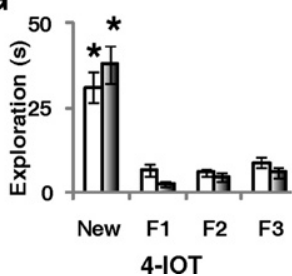

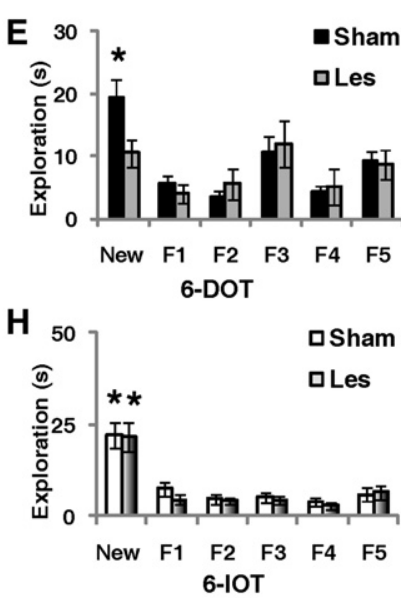

Figure 4. Hippocampus lesion specifically impairs object STM span. Photos of representative Nissl-stained coronal sections of sham $(A)$ and hippocampus lesioned $(B)$ animals. (C-H) New and familiar (F) object exploration during the test phase of the object memory span tasks, in sham and hippocampus lesioned (Les) animals. Hippocampus lesion impaired new object discrimination in the DOT $(C-E)$ performed at a 1-min retention interval, and this effect was dependent on the number of different objects presented; in the 3-DOT and 4-DOT, lesioned animals explored the new object significantly more than all of the familiar objects $(C, D)$; while in the 6-DOT $(E)$, lesioned animals did not prefer the new over the familiar objects. The new object was explored significantly more than all of the other familiar objects in the IOT, independently of the lesion and the number of samples $(F-H) .\left(^{*}\right)$ $P<0.05$ new versus all of the other familiar objects; Duncan post hoc tests.

\section{Mice have a limited object memory span}

How much can our minds remember at a time is a question that has been discussed for many years. The pioneer experimental results of Miller (1956) suggested that humans are only able to hold seven, plus or minus two, digits of information in their short-term memory. However, recent evidence is revising that idea, suggesting that the limit in capacity is lower when adding the limitation of no memory strategies, such as grouping them together (i.e., chunking) (Cowan 2001). In this study, by showing that CD1 male mice have an object memory span of about six objects, we confirm previous findings in monkeys and humans (Luck and Vogel 1997; Levy et al. 2003; Cowan et al. 2005; Heuer and Bachevalier 2011) suggesting that this limited capacity exists. Whether STM has a limited capacity in rodents has been poorly addressed, probably due to the paucity of sensitive and clinically relevant behavioral tasks capable of detecting item memory span impairments in rodents. To our knowledge, a single attempt to develop a memory odor span task has been reported (Dudchenko et al. 2000), which was demonstrated to have a high predictive validity in genetic animal models of Alzheimer's disease (Young et al. 2009; de Rover et al. 2011). In contrast with our results, the results of this study suggest that although there was a although it does not allow any general conclusion of the parietal cortex in this task because the volume of toxin injected only marginally destroyed this area, clearly demonstrates that the deficit we observed in the 6-DOT at 1 min of delay was, indeed, due to the HP lesion and not to the spread of the toxin in the surrounding cortical area (Fig. 6).

Altogether, these findings demonstrate that dorsal HP lesion is as much effective in disrupting object STM in the high memory load condition as it is in disrupting spatial memory.

\section{Discussion}

In this study, we developed a novel behavioral procedure to study object memory span in mice, through which we demonstrated that the object memory span is of about six objects. Using this procedure, we confirmed that selective disruption of the dorsal HP does not affect object STM or LTM when the memory load is low. However, we showed for the first time that excitotoxic lesion of the dorsal HP impairs novel object discrimination in the high memory load condition, and thus reduces the object memory span of mice to four objects. Altogether, these results shed novel light on the role of the HP in item memory and on its potential role in mediating memory span deficit affecting human pathologies, such as aging, Alzheimer's disease, Korsakoff's syndrome, and schizophrenia (Moss et al. 1986; Nilsson 2003; Hill et al. 2010).
Table 2. Mean total objects exploration during the study phase for Experiment 3

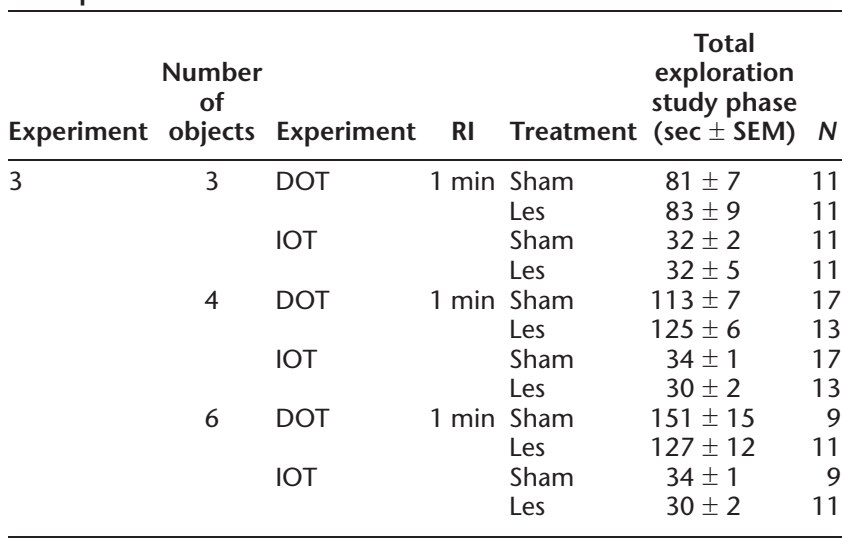

The table reports (from left to right) the experiment to which the data refer, the number of objects used, the kind of task (Different Objects Task [DOT] or Identical Objects Task [IOT]), the retention interval (RI) between the study and the test phase, the treatment the animals received, the total exploration time accumulated during the study phase, the number of subjects per group, and the brain region manipulated for each experiment. The lesion did not significantly affect the total objects exploration during the study phase, as compared with sham. 

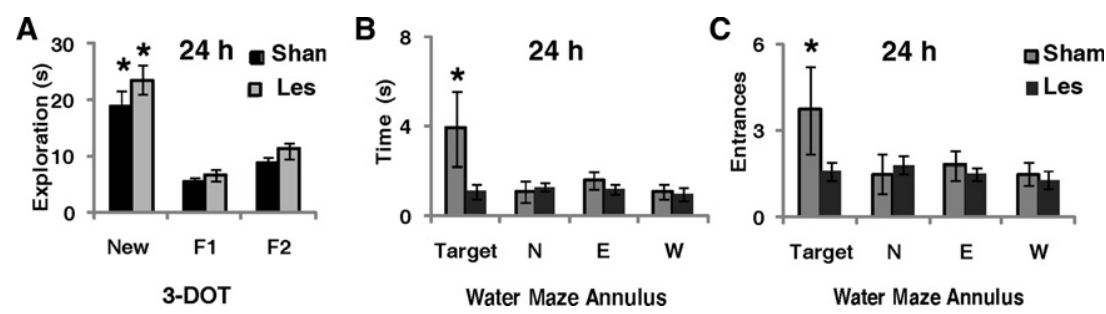

Figure 5. Effects of dorsal hippocampus lesion on long-term object and reference memory. Animals with dorsal hippocampus lesion (Les), similarly to sham animals, explored the new object significantly more than all of the familiar (F) objects during the test phase of the three Different Objects Task performed at a 24-h retention interval $(A)$. Lesioned animals were impaired, as compared with sham animals in the time spent (in seconds) $(B)$ and the number of entrances $(C)$ into the target annulus during the probe test in the water maze task. $\left(^{*}\right) P<0.05$ new versus all of the other familiar objects, or target annulus versus all of the other annulus; Duncan post hoc tests.

span-dependent decrease in the accuracy, performance in the task was always significantly above the chance levels at any span tested (Dudchenko et al. 2000). There are numerous differences between our object span task and the aforementioned study that might explain the different results obtained (e.g., mice vs. rats, object vs. odor; delay: 20 vs. $60 \mathrm{sec}$, etc.). We believe, however, that the main important difference is the way the procedures used in the two studies loaded the working memory system. In this study, we used completely novel (never seen before) and meaningless (not associated to any reward) objects. In contrast, in the odor span task, animals were overtrained on the same set of 25 different scents both before and after the lesion (Dudchenko et al. 2000). Previous studies in monkeys suggested that the memory span is affected by the degree of familiarity of the stimulus (Beason-Held et al. 1999).

Another important difference between the two studies is that while in our task the objects were presented all at once during the study phase, independently of the stimulus set size, so that they shared the same degree of familiarity; in the odor memory span, the animal had to identify a novel stimulus in an increasing array of previously presented or familiar stimuli. This implies that the first stimulus is presented over and over while adding novel stimuli to the array. We believe that the information load in our task is dramatically increased by novel stimuli identity information that has to be processed all at once and maintained during the delay to discriminate the novel object during the test phase. In fact, the DOT, different from the IOT, requires a high degree of identification memory and details discrimination. Based on human studies, the greater the information load of each item in a stimulus class is, the fewer items from that class can be held in memory (Alvarez and Cavanagh 2004). It is important to note that a high degree of identification memory and details discrimination not only can load the working memory system, but it can also recruit recollective-based, instead of familiarity-based, recognition memory mechanisms (Yakovlev et al. 2008).

In conclusion, the DOT presented in this study, different from the odor span task, might rely more on a working memory mechanism and favor the use of recollective-based recognition memory, and thus be better suited to unrevealing working memory span limits in rodents. Accordingly, familiarity recognition in humans seems to be almost limitless even at long retention intervals (Standing 1973), while working memory span has been consistently shown to be limited in humans and monkeys (Luck and Vogel 1997; Beason-Held et al. 1999; Levy et al. 2003, 2004; Shrager et al. 2008; Heuer and Bachevalier 2011). This makes the DOT a good complement to the odor span task for rodents, to study working memory span in mice. et al (2000) using the odor span task.

As we said, however, there are many differences between the two studies, which might explain these different results. In particular, we think that the novelty of the material to be remembered and the higher information load demand in our task, as compared with the odor span task, which proportionally loaded the object working memory system, allowed us to find an effect of HP lesion (Kumaran and Maguire 2007). Furthermore, it has been recently shown that neonatal hippocampus selective lesion in monkeys strongly impairs adulthood novel object recognition in a nonassociative task (incidental learning task based on spontaneous preference for novel objects) (Zeamer et al. 2010), but did not affect it when animals are trained to respond to novelty upon instrumental contingency (Heuer and Bachevalier 2011). This suggests that the nonassociative nature of our task, which makes it quite close to incidental learning in humans and monkeys, might have favored the recruitment of the HP in the object task.

A conservative interpretation of the results of our study suggests that the dorsal HP is not the site where object memory

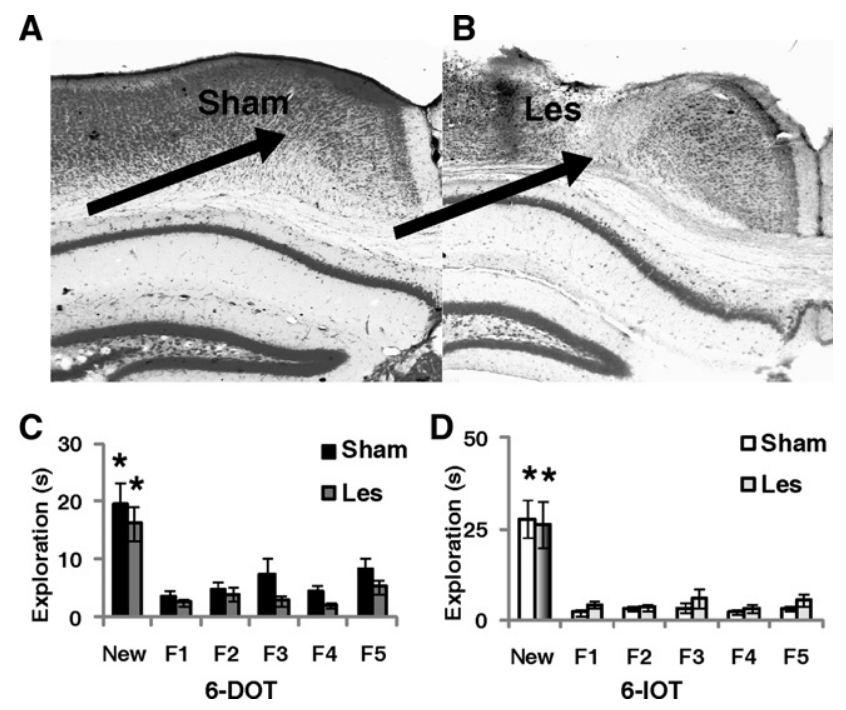

Figure 6. Lesion of the cortex does not affect object short-term memory. Photos of representative Nissl-stained coronal sections of sham animals $(A)$ and cortical lesioned $(B)$ animals. Animals with selective lesion of the cortical region immediately surrounding the dorsal hippocampus (Les), during the test phase of the 6-DOT (C) and 6-IOT (D) object tasks performed at short retention interval $(1 \mathrm{~min})$, explored the new object significantly more than all of the other familiar $(F)$ objects. $\left.{ }^{*}\right) P<0.05$ new versus all of the other familiar objects; Duncan post hoc tests. 
Table 3. Mean total objects exploration during the study phase for Experiments 4 and 6

\begin{tabular}{lcclccc}
\hline $\begin{array}{l}\text { Number } \\
\text { of objects }\end{array}$ & Task & RI & Treatment & $\begin{array}{c}\text { Total } \\
\text { exploration } \\
\text { study phase } \\
\text { (sec } \pm \text { SEM) }\end{array}$ & N & Brain region \\
\hline 3 & DOT & $24 \mathrm{~h}$ & Sham & $70 \pm 5$ & 16 & Hippocampus \\
& & & Les & $73 \pm 7$ & 15 & \\
6 & DOT & 1 min & Sham & $133 \pm 13$ & 10 & Cortex \\
& LOT & & Les & $114 \pm 17$ & 7 & \\
& & & Sham & $32 \pm 2$ & 10 & \\
& & & Les & $34 \pm 0.5$ & 8 & \\
\hline
\end{tabular}

The table reports (from left to right) the number of objects used, the kind of task (Different Objects Task [DOT] or Identical Objects Task [IOT]), the retention interval (RI) between the study and the test phase, the treatment the animals received, the total exploration time accumulated during the study phase, the number of subjects per group, and the brain region manipulated for each experiment. The lesion did not significantly affect the total objects exploration during the study phase, as compared with sham.

representations are formed and stored so that if it is damaged the subject is no longer able to recognize familiar objects and/or form novel object representations whatsoever. However, when the stimulus set size increases, the integrity of the dorsal HP is necessary to discriminate the novel object as compared with familiar ones, suggesting that this brain region is probably specifically recruited to manage a conspicuous amount of information.

The demonstrated role of the HP in novel object discrimination in a condition of high load raises the interesting question of its specific contribution in this process.

According to the memory consolidation theory of the HP, Shrager et al. (2008) suggested that the demonstrated role of the $\mathrm{HP}$ in mediating item memory is dependent on its specific contribution to LTM formation, rather than on working memory maintenance, despite the short-retention time interval. Accumulating experimental evidence suggests that LTM, but not STM, requires de novo proteins synthesis (Klann and Sweatt 2008), a process requiring hours. It is extremely unlikely that a few seconds (in humans) to $11 \mathrm{~min}$ (in our task) are sufficient for this process to occur. Therefore, from a biological point of view, the kind of LTM mechanism that would be activated to support object memory in a high memory load condition at short delay does not overlap the one supporting LTM formation. Furthermore, the fact that animals with dorsal HP lesion were not impaired in the 3-DOT at a 24-h delay suggests that the engagement of an LTM mechanism in object memory tasks is not sufficient to recruit the dorsal HP. In contrast, we believe that being exposed to a high information load is a sufficient condition to recruit the HP in the task performance.

According to the spatial memory theory of the HP, it has been suggested that by offering another source of information (spatialcontextual) to discriminate among multiple overlapping object representations, the HP would be necessary only in high memory conditions, when the ambiguity among multiple possible overlapping representations increases (Cowell et al. 2006). Although in our procedure we controlled for the use of spatial, geometric, and contextual information by not modifying the object position in the arena and by comparing the behavior in the 6-DOT with the one in the 6-IOT containing the same number of items disposed exactly in the same configuration, we cannot rule out this interpretation. A previous study with monkeys, however, made the object's position irrelevant by modifying it between studying and testing (Beason-Held et al. 1999). Contrary to the prediction based on the interpretation of Cowell et al. (2006), they found that even when control animals could not rely on spatial information to solve the task, animals with HP lesion were impaired in object memory load.

The stimuli we used differed in shape, color, size, etc. Increasing the stimulus set size, we increased not only the memory load, but also the stimulus distinctiveness demand, which fits with the view of the HP as selectively involved in recollective-based recognition memory, and/or as a comparator capable of individualizing the representations of highly overlapping inputs (Zeamer et al. 2011), or with the recently suggested role in coordination and use of multiple information streams (Kelemen and Fenton 2010).

\section{Conclusions}

In this study, we developed a novel behavioral procedure to study object memory span. By using it, we showed for the first time that rodents have a limited object memory span that is similar to that of humans (Luck and Vogel 1997). We think that the task we developed is a valuable complement and a necessary alternative to the odor span task recently developed by Dudchenko et al. (2000) for the following reasons: (1) It is the first procedure published to test object span in rodents. (2) It can be used for mice, which are the species most frequently used for gene targeting. (3) It is easy, cheap, and not time-consuming to perform. (4) Since it does not require to overtrain the animals, it allows selective pharmacological manipulation of the different phases (prestudy, post-study phase, etc.), and it allows us to test the acute effect of drugs. (5) We show here that unlike the odor span task, it is as sensitive to HP damage as the water maze task, etc. We think that all of these characteristics represent a major advance in the memory system test armory repertoire.

Using this task, we could shed novel light on the specific contribution of the HP in object STM. Previous findings presented a dichotomous view of spatial and object memory (Lee et al. 2005; Ainge et al. 2006), and between STM and LTM, regarding the role of the dorsal HP. The results we present in this study advocate that the retention interval and the nature of the stimulus are not as important as the stimulus set size itself in recruiting the dorsal HP. We propose that when the subject is confronted with one or a few objects (low load), object-related information from the perirhinal cortex is directly conveyed to the thalamus, bypassing the HP. As an alternative, it might be directed to the dorsal HP, but once there they are not able to activate it. In contrast, being confronted with many different objects, the high information load and/or the high degree of novelty might generate highly convergent inputs that are able to recruit the dorsal HP in the process, and once there they trigger its activation.

An important implication of these results is that the HP might be a common pharmacological target to improve both relational and item memory span deficits when they concurrently occur in pathologies such as Alzheimer's disease and aging.

\section{Materials and Methods}

\section{Subjects}

In all of the experiments of this study, we used outbred CD1 adult (10-16 wk) male mice (Charles River, Italy) housed in groups of three to five subjects. All procedures were performed in strict accordance with the European Communities Council directives and Italian laws on animal care.

\section{Behavioral procedures}

Object memory span task

Animals were isolated for $15 \mathrm{~min}$ in a waiting cage before testing and then subjected to a habituation trial (10 $\mathrm{min})$ in the empty 
testing open field $(35 \times 47 \times 60 \mathrm{~cm})$. After 1 min spent in their waiting cage, they were subjected to the study phase, during which they were allowed to explore the objects. In the first experiment, there were four different experimental groups (see Supplemental Material), each exposed to three, four, six, or nine objects (Fig. 1A-H). Mice were exposed to three, four, six, or nine different objects (Fig. 1A-D). The control task consisted of exposing the mice to an increasing number of identical objects (Fig. 1E-H); therefore, the DOT and IOT were similar (same spatial arrangement, same number of items, etc.) except for the amount of information to be stored (different vs. identical objects). Thus, to avoid any general habituation effect to the task, each animal was submitted only two times to the task, once to the DOT and the other to the IOT of the same stimulus set size $15 \mathrm{~d}$ apart (see Experimental Groups in Supplemental Material), randomizing the order between different animals belonging to the same experimental group (e.g., mouse 1: first to the 3-DOT and $15 \mathrm{~d}$ later to the 3-IOT; mouse 2: first to the 3-IOT and 15 $\mathrm{d}$ later to the 3-DOT, etc.). For the IOT, animals were allowed to accumulate a maximum of $35 \mathrm{sec}$ of total objects exploration, in a 5-min test independently of the number of items. In contrast, for the DOT, we determined the overall study time according to the number of different objects that the animals were exposed to (Fig. 1), setting a 35-sec exploration time for each different object (Fig. 1A-D). A maximum of 10 or $15 \mathrm{~min}$ was given in the 3-DOT, 4-DOT, 6-DOT, and 9-DOT, respectively, to collect the aforementioned exploration times. If the animal did not explore for more than $5 \mathrm{sec}$, it was excluded from the test. Exploration was defined as the time in which the nose was in contact $(<2 \mathrm{~cm}$ from the object) with the object (Broadbent et al. 2004; Ainge et al. 2006). During the test phase, the animal was exposed to identical copies of the familiar objects and one new object. Some of the experiments were performed in different laboratories, in two different cities, and by different experimenters, and the object set was not always identical between experiments, although we tried always to use only two types of novel objects alternated in all conditions. The different experimental conditions were always matched between the experimental and the control groups, and the experimenter was always blind to the treatment the animal received. The position of the novel object was changed across animals in a random order. This makes it extremely unlikely that there was any bias linked to any kind of object used. Retention intervals for STM and LTM were $1 \mathrm{~min}$ and $24 \mathrm{~h}$, respectively. The position of the objects in the field was not modified. The animal's behavior was recorded for $5 \mathrm{~min}$ by a video-tracking system (Any-maze, Stoelting, USA) and analyzed by a trained observer. The most commonly used index of new object discrimination in the spontaneous object recognition task is time spent exploring the new object as a percentage of the total exploration time (Broadbent et al. 2004; Ainge et al. 2006). This measure, however, was not appropriate to compare the performance on tasks presenting one new object and an increasing number of familiar objects. Therefore, new object discrimination was considered to occur in our task when the new object was explored significantly more than all of the other familiar objects present during testing. This is the only method that allows comparison of results of experiments using different object numbers. We distinguished between different degrees of discrimination/ impairment: (1) new object discrimination/no impairment: when the new object was explored significantly more than all the other objects; (2) new object discrimination impairment: when the new object was explored significantly more than some objects but similarly to other objects; and (3) complete lack of new object discrimination: when the new object was explored similarly to all the other objects. Indeed, there were cases in which the within-group comparison did not reveal any impairment (the new object was explored significantly more than all of the familiar objects). Nevertheless, the between-group comparison indicated that the new object in the experimental group was explored significantly less as compared with the new object exploration in the control group. We interpreted this result as preserved new-familiar object discrimination, but impaired new object preference.

\section{Data analysis and statistics}

Mean object exploration during the study phase for all of the experiments was analyzed using one-way ANOVA (Tables 1-3). ANOVA for repeated measures were always performed in all of the experiments we present comparing the different groups reported in the figures (e.g., Experiment 1, comparing different vs. identical task) as between-group variables, and objects (different levels: 3, 4, 6, 9) as repeated measure. However, in all experiments using the object task, to verify whether object discrimination occurred or whether there was impairment, we considered exclusively the results of the Duncan post hoc test comparing the mean exploration time of the new object versus that of all of the other familiar objects within groups. In the graphs, the asterisk symbol $\left.{ }^{*}\right)$ is apposed on the new object to indicate that it was explored significantly more than the entire set of familiar objects. When this condition was not satisfied, the open circle symbol $\left({ }^{\circ}\right)$ is apposed on each of the familiar objects that were explored significantly less as compared with the new object.

\section{Water maze task}

Seven sham and 14 lesioned animals chosen among those tested in the object task were submitted $1 \mathrm{wk}$ after they performed the object task to the water maze task, using exactly the same behavioral procedure that has been previously described for CD1 mice (Sargolini et al. 2003). In brief, the task was performed for three consecutive days. Day 1 (Visible): On the first day $(2$ sessions $\times 2$ trials each), the platform protrudes $1.5 \mathrm{~cm}$ above the surface of the water and is placed in the center of the West (Session 1) and North (Session 2) quadrants. Day 2 (Spatial): The training phase consisted of six sessions of four trials. All of the training sessions were performed as on the previous day, except that the platform was submerged and placed in the center of the South quadrant. Starting points were determined in a pseudorandom order for each session, but the same starting points were used for all the animals. Day 3 (Probe trial): The probe test consisted of one single trial performed without the platform; starting from the center of the pool, mice were allowed $1 \mathrm{~min}$ to search for the platform. The Any-Maze video tracking system recorded the distance (in meters) traveled to reach the platform during the training and the number of entrances and time spent in the different annulus during the probe test.

\section{Statistics}

ANOVA for repeated measures was used to test the effects of treatment ( 2 levels: Les vs. Sham) as the between-group variable, and the entrance and the time in the annulus (4 levels: 4 annuli) for the water maze task as repeated measures. Statistical significance was set at $P<0.05$ for all of the experiments.

\section{Surgery}

\section{Hippocampus lesion}

Anesthetized mice (Avertin; $20 \mu \mathrm{L}$ per gram of mouse body weight, i.p.) were placed in a stereotaxic apparatus (David Kopf Instruments). An injection needle was bilaterally inserted within the HP. The stereotaxic coordinates were $\mathrm{RC}=-1.9 \mathrm{~mm}$, $\mathrm{L}= \pm 1.2 \mathrm{~mm}, \mathrm{DV}=-1.6 \mathrm{~mm}$, according to the mouse brain atlas (Franklin and Paksinos 1997). The needle was connected by plastic tubing to a $2-\mu \mathrm{L}$ Hamilton syringe, which allowed the injection of $0.3 \mu \mathrm{L} /$ side of $20 \mathrm{mg} / \mathrm{mL}$ NMDA (Les) or saline (Sham). Behavioral testing started 10-15 d after surgery. At the end of the behavioral experiments, animals were sacrificed, the brain was removed, and lesion extension was verified under a light microscope by analyzing consecutive coronal brain sections $(50 \mu \mathrm{m})$ stained with Cresyl Violet.

\section{Cortex lesion}

Bilateral lesion of the cortical area surrounding the HP was performed to test whether the behavioral effects observed in the 6-DOT (Different Objects Task) at a short retention interval 
(1 min) in animals with HP lesion was due to a spread of the toxin in the surrounding cortical area. The lesion procedure and lesion extension verification were identical to those described for the $\mathrm{HP}$ - we only changed the DV coordinate from DV $=-1.6$ for the HP to DV $=-0.7$ for the cortex, according to the mouse brain atlas (Franklin and Paxinos 1997).

\section{Acknowledgments}

We thank Elizabeth Illingworth and Pauline Lafenetre for critical revision of the manuscript, and A. Oliverio, R. Gerlai, A. Treves, and G. Diez Roux for critical revision and discussion of a previous version of the manuscript. We also thank R. Andolfi, M. Di Tommaso, and A. Cucciardi for animal care; E. Nusco for technical help; and A.M. Aliperti for language revision. This work was supported by a grant from the Telethon Foundation (http://www. telethon.it) (TEDDSMTELC) and the Alzheimer's Association (http://www.alz.org) to E.D.L. (NIRG-10-173992).

\section{References}

Ainge JA, Heron-Maxwell C, Theofilas P, Wright P, de Hoz L, Wood ER. 2006. The role of the hippocampus in object recognition in rats: Examination of the influence of task parameters and lesion size. Behav Brain Res 167: 183-195.

Alvarez GA, Cavanagh P. 2004. The capacity of visual short-term memory is set both by visual information load and by number of objects. Psychol Sci 15: 106-111.

Beason-Held LL, Rosene DL, Killiany RJ, Moss MB. 1999. Hippocampal formation lesions produce memory impairment in the rhesus monkey. Hippocampus 9: 562-574.

Brebion G. 2003. Working memory, language comprehension, and aging: Four experiments to understand the deficit. Exp Aging Res 29: 269-301.

Broadbent NJ, Squire LR, Clark RE. 2004. Spatial memory, recognition memory, and the hippocampus. Proc Natl Acad Sci 101: 14515-14520.

Brown MW, Aggleton JP. 2001. Recognition memory: What are the roles of the perirhinal cortex and hippocampus? Nat Rev Neurosci 2: 51-61.

Cave CB, Squire LR. 1991. Equivalent impairment of spatial and nonspatial memory following damage to the human hippocampus. Hippocampus 1: $329-340$.

Clatworthy PL, Lewis SJ, Brichard L, Hong YT, Izquierdo D, Clark L, Cools R, Aigbirhio FI, Baron JC, Fryer TD, et al. 2009. Dopamine release in dissociable striatal subregions predicts the different effects of oral methylphenidate on reversal learning and spatial working memory. I Neurosci 29: 4690-4696.

Cowan N. 2001. The magical number 4 in short-term memory: A reconsideration of mental storage capacity. Behav Brain Sci 24: 87-185.

Cowan N, Johnson TD, Saults JS. 2005. Capacity limits in list item recognition: Evidence from proactive interference. Memory 13: 293-299.

Cowell RA, Bussey TJ, Saksida LM. 2006. Why does brain damage impair memory? A connectionist model of object recognition memory in perirhinal cortex. J Neurosci 26: 12186-12197.

de Rover M, Pironti VA, McCabe JA, Acosta-Cabronero J, Arana FS, Morein-Zamir S, Hodges JR, Robbins TW, Fletcher PC, Nestor PJ, et al 2011. Hippocampal dysfunction in patients with mild cognitive impairment: A functional neuroimaging study of a visuospatial paired associates learning task. Neuropsychologia 49: 2060-2070.

Dudchenko PA, Wood ER, Eichenbaum H. 2000. Neurotoxic hippocampal lesions have no effect on odor span and little effect on odor recognition memory but produce significant impairments on spatial span, recognition, and alternation. J Neurosci 20: 2964-2977.

Fischer H, Nyberg L, Karlsson S, Karlsson P, Brehmer Y, Rieckmann A, MacDonald SW, Farde L, Backman L. 2010. Simulating neurocognitive aging: Effects of a dopaminergic antagonist on brain activity during working memory. Biol Psychiatry 67: 575-580.

Franklin K, Paksinos G. 1997. The mouse brain in stereotaxic coordinates. Academic Press, San Diego, CA.

Heuer E, Bachevalier J. 2011. Effects of selective neonatal hippocampal lesions on tests of object and spatial recognition memory in monkeys. Behav Neurosci 125: 137-149.
Hill SK, Griffin GB, Miura TK, Herbener ES, Sweeney JA. 2010. Salience of working-memory maintenance and manipulation deficits in schizophrenia. Psychol Med 40: 1979-1986.

Hunsaker MR, Fieldsted PM, Rosenberg JS, Kesner RP. 2008. Dissociating the roles of dorsal and ventral CA1 for the temporal processing of spatial locations, visual objects, and odors. Behav Neurosci 122: 643-650.

Kelemen E, Fenton AA. 2010. Dynamic grouping of hippocampal neural activity during cognitive control of two spatial frames. PLoS Biol 8: e1000403. doi: 10.1371/journal.pbio. 1000403.

Klann E, Sweatt JD. 2008. Altered protein synthesis is a trigger for long-term memory formation. Neurobiol Learn Mem 89: 247-259.

Kumaran D, Maguire EA. 2007. Which computational mechanisms operate in the hippocampus during novelty detection? Hippocampus 17: $735-748$.

Lee I, Hunsaker MR, Kesner RP. 2005. The role of hippocampal subregions in detecting spatial novelty. Behav Neurosci 119: 145-153.

Lee EY, Cowan N, Vogel EK, Rolan T, Valle-Inclan F, Hackley SA. 2010. Visual working memory deficits in patients with Parkinson's disease are due to both reduced storage capacity and impaired ability to filter out irrelevant information. Brain 133: 2677-2689.

Levy DA, Manns JR, Hopkins RO, Gold JJ, Broadbent NJ, Squire LR. 2003. Impaired visual and odor recognition memory span in patients with hippocampal lesions. Learn Mem 10: 531-536.

Levy DA, Hopkins RO, Squire LR. 2004. Impaired odor recognition memory in patients with hippocampal lesions. Learn Mem 11: 794-796.

Luck SJ, Vogel EK. 1997. The capacity of visual working memory for features and conjunctions. Nature 390: 279-281.

Miller G. 1956. The magical number seven plus or minus two: Some limits on our capacity for processing information. Psychol Rev 2: 81-97.

Moss MB, Albert MS, Butters N, Payne M. 1986. Differential patterns of memory loss among patients with Alzheimer's disease, Huntington's disease, and alcoholic Korsakoff's syndrome. Arch Neurol 43: 239-246.

Nilsson LG. 2003. Memory function in normal aging. Acta Neurol Scand 179: $7-13$.

Sargolini F, Florian C, Oliverio A, Mele A, Roullet P. 2003. Differentia involvement of NMDA and AMPA receptors within the nucleus accumbens in consolidation of information necessary for place navigation and guidance strategy of mice. Learn Mem 10: 285-292.

Shrager Y, Levy DA, Hopkins RO, Squire LR. 2008. Working memory and the organization of brain systems. J Neurosci 28: 4818-4822.

Standing L. 1973. Learning 10,000 pictures. Q J Exp Psychol 25: 207-222.

Steckler T, Drinkenburg WH, Sahgal A, Aggleton JP. 1998. Recognition memory in rats-I. Concepts and classification. Prog Neurobiol 54: $289-311$.

Vago DR, Kesner RP. 2008. Disruption of the direct perforant path input to the CA1 subregion of the dorsal hippocampus interferes with spatial working memory and novelty detection. Behav Brain Res 189: $273-283$.

van Vugt MK, Schulze-Bonhage A, Litt B, Brandt A, Kahana MJ. 2010. Hippocampal gamma oscillations increase with memory load. J Neurosci 30: 2694-2699.

Wan RQ, Pang K, Olton DS. 1994. Hippocampal and amygdaloid involvement in nonspatial and spatial working memory in rats: Effects of delay and interference. Behav Neurosci 108: 866-882.

Wible CG, Shiber JR, Olton DS. 1992. Hippocampus, fimbria-fornix, amygdala, and memory: Object discriminations in rats. Behav Neurosci 106: $751-761$.

Yakovlev V, Amit DJ, Romani S, Hochstein S. 2008. Universal memory mechanism for familiarity recognition and identification. J Neurosci 28: $239-248$.

Young JW, Sharkey J, Finlayson K. 2009. Progressive impairment in olfactory working memory in a mouse model of Mild Cognitive Impairment. Neurobiol Aging 30: 1430-1443.

Zeamer A, Heuer E, Bachevalier J. 2010. Developmental trajectory of object recognition memory in infant rhesus macaques with and without neonatal hippocampal lesions. J Neurosci 30: 9157-9165.

Zeamer A, Meunier M, Bachevalier J. 2011. Stimulus similarity and encoding time influence incidental recognition memory in adult monkeys with selective hippocampal lesions. Learn Mem 18: $170-180$.

Received December 7, 2011; accepted in revised form March 8, 2012. 


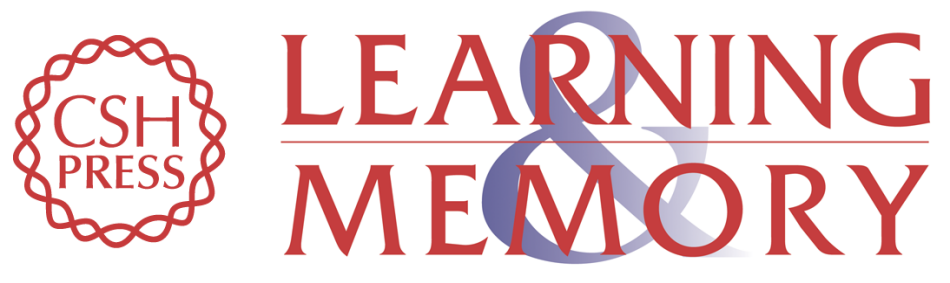

\section{Role of the dorsal hippocampus in object memory load}

Sara Sannino, Fabio Russo, Giulia Torromino, et al.

Learn. Mem. 2012, 19:

Access the most recent version at doi:10.1101//m.025213.111

Supplemental http://learnmem.cshlp.org/content/suppl/2012/04/20/19.5.211.DC1

References This article cites 40 articles, 12 of which can be accessed free at: http://learnmem.cshlp.org/content/19/5/211.full.html\#ref-list-1

License

Email Alerting Receive free email alerts when new articles cite this article - sign up in the box at the Service top right corner of the article or click here. 